Quim. Nova, Vol. 36, No. 7, 972-977, 2013

\title{
AVALIAÇÃO DE TRATAMENTOS DA AMOSTRA EM MICROESCALA PARA A DETERMINAÇÃO DE K, Mg, Na E Zn EM CARNES POR TÉCNICAS DE ESPECTROMETRIA ATÔMICA
}

\author{
Camila Mizette Oliz, Camila Corrêa Pereira, Mariana Antunes Vieira, Anderson Schwingel Ribeiro e Adriane Medeiros \\ Nunes* \\ Centro de Ciências Químicas, Farmacêuticas e de Alimentos, Universidade Federal de Pelotas, 96160-000 Capão do Leão - RS, Brasil
}

Recebido em 28/9/12; aceito em 19/2/13; publicado na web em 15/4/13

\begin{abstract}
EVALUATION OF SAMPLE TREATMENT IN MICRO-SCALE FOR THE DETERMINATION OF K, Mg, Na AND Zn IN MEAT BY ATOMIC SPECTROMETRY TECHNIQUES. This paper describes the development of methods in micro-scale for the determination of $\mathrm{K}, \mathrm{Mg}, \mathrm{Na}$ and $\mathrm{Zn}$ in meat by atomic spectrometry techniques. The limits of detection (LOD) for $\mathrm{K}$ and $\mathrm{Na}$ by microdigestion were 0.18 and $0.20 \mathrm{mg} \mathrm{g}^{-1}$, respectively whereas LOD for $\mathrm{Mg}$ and $\mathrm{Zn}$ by microsolubilization with TMAH were 2.40 and $18.4 \mu \mathrm{g} \mathrm{g}^{-1}$, respectively. The RSD values were lower than $6.0 \%$ and the CRMs analyzed showed values with $95 \%$ agreement. The proposed methods are simple, fast and use small amounts of sample (around $10 \mathrm{mg}$ ) yet do not require special equipment for sample preparation.
\end{abstract}

Keywords: meat; sample preparation; atomic spectrometry techniques.

\section{INTRODUÇÃO}

As carnes, em geral, são fontes importantes de proteínas, lipídios, vitaminas lipossolúveis e minerais como $\mathrm{Zn}$ e $\mathrm{Mg}$, os quais são considerados minerais essenciais para o organismo humano, exercendo funções importantes como a participação em várias reações celulares e também são requeridos como cofatores em diversos sistemas enzimáticos dos processos metabólicos. ${ }^{1}$

Contudo, atualmente, tem-se evidenciado um crescimento do mercado de produtos processados à base de carne, já que a produção de alimentos de fácil preparo se faz cada vez mais necessária, visto o ritmo acelerado da vida cotidiana. Entretanto, no decorrer do processamento industrial, a disponibilidade, bem como perdas e ganhos de nutrientes essenciais pode ser afetada, o que irá alterar a qualidade nutricional do alimento. ${ }^{2-4}$

Além disso, a conservação das carnes, bem como de outros alimentos processados, normalmente é feita pela adição de sais de $\mathrm{Na}$, principalmente o cloreto de sódio $(\mathrm{NaCl})$, já que este atua na minimização da ação de agentes naturais (enzimas ou micro-organismos), aumentando a vida de prateleira dos alimentos. ${ }^{5,6}$

Tendo em vista que, quantidades excessivas de $\mathrm{Na}$ estão sendo associadas ao desenvolvimento de doenças cardiovasculares, iniciativas estão sendo feitas pelo Brasil, bem como por parte dos principais países, para redução da adição de $\mathrm{NaCl}$ nos alimentos. Em contrapartida, como o $\mathrm{K}$ atua de forma efetiva em conjunto com o $\mathrm{Na}$ para manter o equilíbrio hídrico do organismo humano, facilitando as perdas de águas que estão diretamente associadas à eliminação de íons $\mathrm{Na}^{+}$, um aumento das concentrações de $\mathrm{K}$ na dieta pode proteger contra a hipertensão. Deste modo, sabe-se que o ideal seria encontrar quantidades semelhantes de $\mathrm{Na}$ e $\mathrm{K}$ nos alimentos em geral. ${ }^{7-9}$

$\mathrm{O}$ controle de metais como $\mathrm{Na}, \mathrm{K}, \mathrm{Mg}$ e $\mathrm{Zn}$, bem como os principais nutrientes contidos nos alimentos cárneos, é de suma importância para garantir a qualidade nutricional e toxicológica destes produtos. Uma vez que os métodos convencionais de análise são em sua maioria morosos e mais propensos a contaminações, faz-se necessário o desenvolvimento de métodos rápidos e simples, com a

*e-mail: adriane.mn@hotmail.com utilização de pequenas quantidades de amostra e reagente, gerando uma quantidade menor de resíduo e, principalmente, fornecendo resultados exatos e precisos..$^{10,11}$

Dentre os métodos convencionais de preparo de amostras destacam-se a digestão ácida em sistemas abertos, os quais necessitam de quantidades relativamente elevadas de ácidos ou misturas de ácidos, bem como de um tempo de análise maior, o que torna a análise morosa e dispendiosa. ${ }^{10-12}$ Sendo assim, vários métodos alternativos de preparação das amostras estão sendo desenvolvidos com o intuito de minimizar possíveis inconvenientes relacionados a esta etapa. Como exemplo, pode-se destacar a amostragem por suspensão, a qual poderá envolver a solubilização parcial da matriz da amostra, utilizando tanto reagentes ácidos como alcalinos. ${ }^{12-14}$ Processos de microdigestão também vêm sendo estudados, os quais caracterizam-se por utilizar uma massa menor de amostra e um menor tempo de exposição das amostras na presença de quantidades reduzidas de reagentes. ${ }^{15,16}$

Assim, o objetivo principal deste trabalho foi de avaliar métodos de preparo de amostras de carnes (frescas e processadas) em microescala, baseados em procedimentos de microdigestões e microssolubilizações, os quais constituem alternativas interessantes e viáveis, pois são realizados em um curto período de tempo, além de utilizar quantidades mínimas de amostras e reagentes para determinação de $\mathrm{K}, \mathrm{Mg}, \mathrm{Na}$ e $\mathrm{Zn}$ por técnicas de espectrometria atômica.

\section{PARTE EXPERIMENTAL}

\section{Equipamentos e parâmetros instrumentais}

Um espectrômetro de absorção atômica modelo AA-6300 (Shimadzu, Japão) com chama ar-acetileno e corretor de fundo SmithHieftje foi utilizado para a determinação de $\mathrm{Na}$ e K por espectrometria de absorção atômica com chama em amostras comerciais de carnes processadas e materiais de referência certificados (CRMs). Para as determinações de $\mathrm{Mg}$ e $\mathrm{Zn}$ foi utilizado um corretor de fundo com lâmpada de arco de deutério e as análises para estes elementos foram feitas nas mesmas amostras e adicionalmente em duas amostras de carnes frescas. Foram utilizadas lâmpadas de cátodo oco (Hamamatsu Photonics K.K, Japão), para cada elemento e empregadas as condições operacionais recomendadas pelo fabricante do equipamento: 
comprimento de onda de $589,0 \mathrm{~nm}, 766,5 \mathrm{~nm}, 285,2 \mathrm{~nm}$ e $213,9 \mathrm{~nm}$ e corrente da lâmpada: 8/600, 8/600, 8/0 e 8/0 mA para Na, K, Mg e $\mathrm{Zn}$, respectivamente. Todas as análises foram feitas utilizando-se um acessório disponível nesse modelo de espectrômetro, o qual permite a microinjeção de pequenos volumes de solução da amostra na chama ( 5 a $200 \mu \mathrm{L}$ ). Esse sistema resulta em um sinal transiente, podendo ser registrada tanto a altura máxima do pico, quanto a sua área integrada. As determinações de $\mathrm{Na}$ e $\mathrm{K}$ também foram realizadas em um fotômetro de chama modelo B462 (Micronal, São Paulo, SP, Brasil), o qual foi operado nas seguintes condições para ambos os elementos: taxa de aspiração da amostra $\left(5 \mathrm{~mL} \mathrm{~min}^{-1}\right)$, ar comprimido $\left(9 \mathrm{~L} \mathrm{~min}^{-1}\right)$ a uma pressão de $1 \mathrm{kgf} \mathrm{cm}^{-2}$ e chama de gás butano.

As amostras foram cortadas com faca inox para evitar contaminação e, posteriormente, homogeneizadas utilizando um miniprocessador de alimentos (Black \& Decker, Brasil) e pesadas utilizando uma balança analítica com resolução de $0,1 \mathrm{mg}$ e tara máxima de $210 \mathrm{~g}$ (Ohaus Adventurer, Modelo AR 2140, Pine Brook, NJ, EUA). Para a microdigestão ácida das amostras foi utilizado um forno de micro-ondas (Panasonic, Manaus, AM, Brasil, Modelo NN-ST571WRU de frequência de $2450 \mathrm{MHz}$ ) e para a solubilização em meio de ácido fórmico foi utilizado um banho ultrassônico (Modelo Q335D, Quimis, SP, Brasil).

\section{Reagentes e soluções}

Todos os reagentes utilizados foram de grau analítico. A água utilizada no preparo das amostras e das soluções foi obtida por um sistema de purificação de água Direct-Q 3 (Millipore Corporation, Bedford, MA, E.U.A.), com resistividade de 18,3 $\mathrm{M} \Omega \mathrm{cm}^{-1}$. Ácido nítrico $65 \%(\mathrm{~m} / \mathrm{m})$ (Synth, Brasil) foi purificado por destilação em um sistema de sub-ebulição de quartzo MA-075 (Marconi, Piracicaba, SP, Brasil). Soluções padrões de K, Mg, Na e Zn foram diariamente preparadas a partir da diluição de uma solução estoque contendo $1000 \mathrm{mg} \mathrm{L}^{-1}$ (Fluka, Buchs, Alemanha) de cada elemento em água deionizada, bem como as soluções $(\mathrm{CsCl}$ para $\mathrm{Na}$ e $\mathrm{K}$ e $\mathrm{KCl}$ para $\mathrm{Mg}$ ) utilizadas para a minimização de interferências relacionadas ao processo de atomização. Os seguintes reagentes foram utilizados para a microssolubilização das amostras: ácido fórmico (Fluka Analítica, Alemanha), hidróxido de tetrametilamônio pentahidratado ou TMAH (Sigma Aldrich, Alemanha) e, para a microdigestão foi utilizado o ácido nítrico (Synth, Brasil), previamente purificado, como já citado.

Os seguintes CRMs foram utilizados para o desenvolvimento e verificação da exatidão das metodologias propostas para a determinação de Na e K: CRM 1546 (carne homogênea), CRM 1577c (fígado bovino). Já para as determinações de $\mathrm{Mg}$ e $\mathrm{Zn}$, foram empregados o CRM 1577c e CRM 1577b, ambos de fígado bovino. Os CRMs utilizados são produzidos pelo NIST- National Institute of Standards and Technology (Gaithersburg, MD, USA).

Vidrarias e recipientes de plásticos, como ponteiras de micropipetas, tubos de centrífuga e copos pertencentes ao amostrador automático do equipamento foram imersos em um banho de $\mathrm{HNO}_{3}$ $10 \%$ (v/v), por no mínimo 48 horas e, posteriormente, lavados com água deionizada e secos em ambiente fechado.

\section{Preparo das amostras}

As amostras utilizadas na avaliação das metodologias propostas foram de origem comercial, provenientes de diferentes fabricantes (salsicha, almôndega e carne bovina fatiada) e também frescas (bovinas e suínas). Para este estudo, quantidades significativas de amostras ( 200 g ou todo o enlatado) foram inicialmente cortadas com uma faca inox, moídas simultaneamente e homogeneizadas com auxílio de um miniprocessador de alimentos, sem tempo fixo, até as amostras se apresentarem suficientemente homogêneas, de acordo com a sua natureza e quantidade. Após esta etapa, os patês das amostras foram preservados em pequenas porções sob refrigeração, a uma temperatura de $-16{ }^{\circ} \mathrm{C}$ em potes de plástico, previamente descontaminados. As amostras foram descongeladas para análise à temperatura ambiente $\mathrm{e}$ homogeneizadas. Após este procedimento, uma alíquota foi retirada e o restante da amostra foi novamente congelado. As amostras foram preparadas utilizando-se diferentes procedimentos para determinação de $\mathrm{Na}$ e K ; e para Mg e Zn os quais serão descritos, respectivamente, nos itens a seguir.

\section{Preparo das amostras para determinação de Na e K}

As amostras reais (carnes processadas) e os CRMs citados anteriormente foram tratadas a partir de três diferentes procedimentos de preparo, descritos abaixo. Os procedimentos 2 e 3 foram usados para verificar a exatidão do método de microdigestão proposto.

\section{Procedimento 1 - Microdigestão (Mineralização com $\mathrm{HNO}_{3}$ em sistema fechado)}

As amostras foram preparadas diretamente em frascos volumétricos de polipropileno (PP) de $50 \mathrm{~mL}$. Foram pesadas aproximadamente $10 \mathrm{mg}$ de amostra de carne processada e adicionados $150 \mu \mathrm{L}$ de $\mathrm{HNO}_{3}$ $65 \%(\mathrm{~m} / \mathrm{m})$. A mistura foi levada a um forno de micro-ondas, onde primeiramente foi realizada a otimização da potência e o tempo necessário para obter uma digestão completa das amostras. Posteriormente, as amostras foram colocadas uma a uma no centro do prato giratório do forno, como sugerido por Rosini et al. ${ }^{17}$ os quais evidenciaram que este é o local de melhor distribuição da radiação micro-ondas. No forno, as amostras permaneceram por 5 minutos na potência máxima determinada (680 Watts). A digestão foi considerada completa quando toda a gordura da amostra de carne foi dissolvida e a solução resultante mostrou-se homogênea e límpida. Após o resfriamento, as amostras foram aferidas a $50 \mathrm{~mL}$ com água deionizada.

A aplicabilidade do procedimento de microdigestão só pode ser avaliada nas amostras de carnes para determinação de Na e K, já que esses minerais se apresentam em altas concentrações em produtos processados e assim, necessitam de um alto fator diluição, permitindo o uso de frascos com uma maior capacidade volumétrica, o que faz com que estes sejam aptos a garantir a total solubilização dos gases formados durante as reações de mineralização. Cabe salientar que o uso deste procedimento para determinação de elementos que se encontram em menores concentrações (como por exemplo, $\mathrm{Mg}$ e $\mathrm{Zn}$ ), necessita de uma melhor otimização das condições de trabalho, aliada a utilização de um sistema que permita a digestão das amostras sob radiação micro-ondas associado ao emprego de micro frascos, garantindo a eficiência das microdigestões, bem como a segurança do analista.

\section{Procedimento 2 - Microssolubilização com TMAH}

As amostras foram preparadas através de uma simples mistura de aproximadamente $10 \mathrm{mg}$ de amostra com $150 \mu \mathrm{L}$ da solução de TMAH $25 \%(\mathrm{~m} / \mathrm{v})$, as quais foram preparadas diretamente nos frascos volumétricos de polipropileno de $50 \mathrm{~mL}$. As amostras ficaram em contato com a solução alcalina durante um período de aproximadamente 12 horas em temperatura ambiente e o frasco permaneceu fechado até a completa solubilização. Após, o volume foi completado a $50 \mathrm{~mL}$ com água deionizada e a concentração final da solução foi de $0,075 \%$ $(\mathrm{m} / \mathrm{v})$ de TMAH. A suspensão resultante foi submetida para análise.

\section{Procedimento 3 - Microssolubilização com ácido fórmico ( $\mathrm{HCOOH})$}

Amostras de carne foram solubilizadas em meio de $\mathrm{HCOOH}$ 
segundo o método descrito por Scriver et al, ${ }^{18}$ porém, a metodologia foi adaptada ao sistema em microescala: aproximadamente $10 \mathrm{mg}$ de amostra foram pesados diretamente em frascos de polipropileno de $50 \mathrm{~mL}$, seguido da adição de $200 \mu \mathrm{L}$ de HCOOH. Em seguida, a mistura foi colocada em um banho de ultrassom a $70{ }^{\circ} \mathrm{C}$ por um período de 2-3 h. Após o resfriamento, o volume foi completado a 50 $\mathrm{mL}$ com água deionizada para as subsequentes análises.

\section{Preparo das amostras para determinação de Mg e Zn}

Para a determinação de $\mathrm{Mg}$ e $\mathrm{Zn}$ foram estudados dois procedimentos de preparo de amostra: microssolubilização usando o meio ácido $(\mathrm{HCOOH})$ ou o meio alcalino (TMAH). Quantidades diferentes de amostras foram necessárias para determinação de $\mathrm{Mg}$ e $\mathrm{Zn}$, conforme descrito a seguir nos procedimentos 4 e 5 :

\section{Procedimento 4 - Determinação de $\mathrm{Mg}$}

As amostras foram preparadas diretamente em frascos volumétricos do tipo eppendorf de $2 \mathrm{~mL}$. Tanto as massas de amostras pesadas quanto os volumes adicionados de TMAH e $\mathrm{HCOOH}$, assim como o tempo necessário para ocorrer a completa solubilização foram iguais aos procedimentos 2 e 3 para ambos os procedimentos $(4 \mathrm{e}$ 5), descritos anteriormente. Após a solubilização as amostras foram aferidas a $2 \mathrm{~mL}$ com água deionizada para posterior análise.

\section{Procedimento 5 - Determinação de $\mathrm{Zn}$}

Tendo em vista que o $\mathrm{Zn}$ normalmente se apresenta em baixas concentrações em carnes, massas distintas de amostras reais foram pesadas, as quais foram dependentes da natureza da amostra a ser analisada. Para as amostras de almôndegas, carne bovina processada enlatada e carne bovina fresca foram pesados aproximadamente 20 mg. Já para as amostras de carne suína fresca e salsicha foi necessário pesar uma massa de aproximadamente $50 \mathrm{mg}$. As solubilizações destas respectivas massas em meio de TMAH e $\mathrm{HCOOH}(150 \mu \mathrm{L}$ e $400 \mu \mathrm{L}$, respectivamente) foram feitas diretamente nos frascos volumétricos do tipo eppendorf de $2 \mathrm{~mL}$ e, após a completa solubilização, as amostras foram aferidas a este volume com água deionizada para posterior análise.

\section{Metodologia}

Primeiramente, estudos foram feitos com o objetivo de verificar possíveis erros de pesagem, bem como calcular o teor de umidade referente a cada amostra analisada. Para tanto, aproximadamente $500 \mathrm{mg}$ de amostras de carne (processadas e frescas) foram pesadas em um vidro de relógio, secas em uma estufa a uma temperatura de aproximadamente $100{ }^{\circ} \mathrm{C}$ e mantidas até adquirirem peso constante, eliminando assim, a umidade.

Um estudo adicional de calibração em meio aquoso foi realizado para todos os elementos a fim de avaliar se as curvas poderiam ser feitas nestas condições. No entanto, os resultados analíticos foram obtidos pelo preparo das curvas de calibração usando soluções preparadas no mesmo meio utilizado para o tratamento das amostras, já que as curvas obtidas em meio aquoso não apresentaram sensibilidades semelhantes. Todas as amostras, quando necessário, foram diluídas com água deionizada, a fim de se adequarem à faixa linear de calibração. Cabe salientar que todos os resultados obtidos, tanto para as amostras de carnes quanto para os CRMs, foram calculados baseados na massa úmida de cada amostra.

Para determinação de $\mathrm{Na}$ e K por FAAS foi necessária a adição de um tampão de ionização $\mathrm{CsCl}$ a $0,1 \%(\mathrm{~m} / \mathrm{v})$, concentração esta indicada pelo fabricante, a qual foi adicionada nas soluções das amostras e padrões para minimizar interferências de ionização. ${ }^{19}$ Para as determinações de $\mathrm{Mg}$ por FAAS foi utilizado o $\mathrm{KCl}\left(10 \mathrm{mg} \mathrm{L}^{-1}\right)$ nas soluções das amostras e padrões, pois a solução recomendada pelo fabricante $\left(\mathrm{LaCl}_{3} 0,1 \%(\mathrm{~m} / \mathrm{v})\right)$ não pôde ser usada em meio de TMAH devido à formação de precipitado que causou o entupimento do sistema de nebulização.

Para todas as análises foi utilizado um volume de $200 \mu \mathrm{L}$ de solução de amostra e padrões, que foi introduzido diretamente no sistema de nebulização. O sinal transiente foi avaliado pela área do pico integrada, o que resultou em um ganho de sensibilidade.

\section{RESULTADOS E DISCUSSÃO}

\section{Determinação de Na e K em amostras de carnes processadas}

Embora a metodologia proposta para determinação de Na e K em amostras de carnes tenha se baseado no princípio da metodologia recomendada pelo Ministério da Agricultura para análise de carnes, ${ }^{20}$ como por exemplo, o uso da digestão ácida na presença de $\mathrm{HNO}_{3}$ sob aquecimento, as metodologias propostas aqui inseriram mudanças significativas em prol, principalmente, da Química Analítica moderna e dos princípios da Química Verde. Entre elas se destacam o uso de quantidades reduzidas de amostras e, consequentemente, de reagentes, bem como a praticidade aliada à segurança da análise, com uma menor manipulação da amostra e o tempo de análise consideravelmente reduzido.

É importante salientar também que as microdigestões foram realizadas em um sistema fechado, utilizando aquecimento por radiação micro-ondas, de baixo custo, dispensando sistemas de controle de temperatura e pressão, uma vez que a análise não apresenta riscos de acidentes, já que quantidades mínimas de amostras e reagentes são utilizadas, assegurando assim uma digestão completa da amostra e a integridade física do analista. Também facilita a popularização da utilização deste procedimento em análises de rotina.

De acordo com os resultados apresentados na Tabela 1, foi possível verificar que o procedimento proposto baseado na microdigestão ácida (procedimento 1), aplicado à análise de três amostras reais de carnes, para a determinação de Na e K por diferentes técnicas (FAAS e FAES), se mostrou eficaz, uma vez que não houve diferenças significativas entre os resultados a um nível de confiança de $95 \%$ pela aplicação do teste $t$-student pareado.

Posteriormente, conforme apresentado na Tabela 2, a verificação da exatidão desta metodologia também foi avaliada com base na análise das mesmas amostras de carnes preparadas em diferentes meios (TMAH e $\mathrm{HCOOH}$ ) conforme anteriormente descrito nos procedimentos 2 e 3 . As concentrações obtidas pelos diferentes métodos de preparo das amostras estão de acordo para os analitos em estudo, indicando a exatidão dos resultados, atestado pela aplicação do teste $t$-student pareado.

Com este mesmo objetivo, diferentes amostras de material biológico certificadas foram analisadas para determinação de $\mathrm{Na}$ e K por FAAS e FAES. Com base nos resultados apresentados na Tabela 3, foi verificado que os valores de concentração para Na e K obtidos para os CRMs estudados foram satisfatórios, com aproximadamente $95 \%$ de concordância entre os valores encontrados e os valores certificados. Cabe ressaltar que os CRMs analisados são considerados adequados para representar as amostras reais de carnes, bem como atestar a homogeneidade das mesmas, principalmente o CRM 1546, o qual é produzido como uma amostra real de carne, contendo a umidade semelhante das amostras reais utilizada neste trabalho, porém, com as concentrações dos analitos certificadas.

Avaliando-se as concentrações obtidas para os analitos em estudo, nas três amostras de carnes processadas analisadas (Tabelas 1 e 2), pôde-se atestar que as concentrações encontradas de $\mathrm{Na}$ foram 
Tabela 1. Valores de concentração obtidos de Na e K por FAES e FAAS (média \pm desvio padrão; n=3) em amostras de carnes processadas preparadas pelo processo de microdigestão em meio de $\mathrm{HNO}_{3}$

\begin{tabular}{|c|c|c|c|c|c|c|c|}
\hline \multirow{2}{*}{ Analito } & \multirow{2}{*}{ Amostra } & \multicolumn{3}{|l|}{ F AES } & \multicolumn{3}{|l|}{ F AAS } \\
\hline & & $\mathrm{VE}\left(\mathrm{mg} \mathrm{kg}^{-1}\right)$ & RSD (\%) & $\operatorname{LOD}\left(\mathrm{mg} \mathrm{g}^{-1}\right)$ & $\mathrm{VE}\left(\mathrm{mg} \mathrm{kg}^{-1}\right)$ & RSD (\%) & $\operatorname{LOD}\left(\mathrm{mg} \mathrm{g}^{-1}\right)$ \\
\hline \multirow{3}{*}{ K } & A & $1.363,5 \pm 67,8$ & 4,9 & \multirow{3}{*}{0,18} & $1.128,5 \pm 51,6$ & 4,6 & \multirow{3}{*}{1,09} \\
\hline & B & $958,2 \pm 37,1$ & 3,9 & & $1.117,3 \pm 50,9$ & 4,6 & \\
\hline & $\mathrm{C}$ & $1.687,9 \pm 88,5$ & 5,2 & & $1.752,7 \pm 22,2$ & 1,3 & \\
\hline \multirow{3}{*}{$\mathrm{Na}$} & A & $5.780,2 \pm 237,2$ & 4,1 & \multirow{3}{*}{0,20} & $5.607,9 \pm 117,6$ & 2,1 & \multirow{3}{*}{1,30} \\
\hline & B & $8.859,7 \pm 76,9$ & 0,9 & & $8.393,9 \pm 214,9$ & 2,6 & \\
\hline & $\mathrm{C}$ & $2.952,2 \pm 9,7$ & 0,3 & & $2.585,3 \pm 79,3$ & 3,1 & \\
\hline
\end{tabular}

A: Carne Bovina Processada; B: Salsicha Vienna; C: Almôndega. VE: Valor Encontrado; RSD: Desvio padrão relativo; LOD: Limite de Detecção.

Tabela 2. Valores de concentração obtidos para Na e K por FAES em amostras de carnes processadas preparadas em dois diferentes meios (média \pm desvio padrão; $n=3$ )

\begin{tabular}{|c|c|c|c|c|c|c|c|}
\hline \multirow{2}{*}{ Analito } & \multirow{2}{*}{ Amostra } & \multicolumn{3}{|c|}{ ТМАH } & \multicolumn{3}{|c|}{ Ácido Fórmico } \\
\hline & & $\mathrm{VE}\left(\mathrm{mg} \mathrm{kg}^{-1}\right)$ & $\operatorname{RSD}(\%)$ & $\operatorname{LOD}\left(\mathrm{mg} \mathrm{g}^{-1}\right)$ & $\operatorname{VE}\left(\mathrm{mg} \mathrm{kg}^{-1}\right)$ & $\operatorname{RSD}(\%)$ & $\operatorname{LOD}\left(\mathrm{mg} \mathrm{g}^{-1}\right)$ \\
\hline \multirow{3}{*}{ K } & A & $1.259,0 \pm 97,4$ & 7,7 & \multirow{3}{*}{0,12} & $1.297,4 \pm 10,9$ & 0,8 & \multirow{3}{*}{0,03} \\
\hline & B & $1.048,1 \pm 65,5$ & 6,2 & & $1.140,7 \pm 77,5$ & 6,8 & \\
\hline & $\mathrm{C}$ & $1.437,4 \pm 44,3$ & 3,1 & & $1.675,4 \pm 16,9$ & 1,0 & \\
\hline \multirow{3}{*}{$\mathrm{Na}$} & A & $5.367,8 \pm 36,9$ & 0,7 & \multirow{3}{*}{0,14} & $5.162,5 \pm 241,6$ & 4,7 & \multirow{3}{*}{0,05} \\
\hline & B & $8.751,6 \pm 83,4$ & 1,0 & & $8.569,4 \pm 132,2$ & 1,5 & \\
\hline & $\mathrm{C}$ & $2.860,4 \pm 61,9$ & 2,2 & & $2.904,3 \pm 74,9$ & 2,6 & \\
\hline
\end{tabular}

A: Carne Bovina Processada; B: Salsicha Vienna; C: Almôndega. VE, Valor Encontrado; RSD, Desvio Padrão Relativo; LOD, Limite de Detecção.

Tabela 3. Valores de concentração de Na e K encontrados nos CRMs por FAES e FAAS (média \pm desvio padrão, n=3) através do processo de microdigestão com $\mathrm{HNO}_{3}$

\begin{tabular}{|c|c|c|c|c|c|}
\hline \multirow{2}{*}{ Amostra } & \multirow{2}{*}{$\mathrm{VC}$} & \multicolumn{2}{|c|}{ F AES } & \multicolumn{2}{|c|}{ F AAS } \\
\hline & & VE & $\operatorname{RSD}(\%)$ & VE & $\operatorname{RSD}(\%)$ \\
\hline \multicolumn{6}{|c|}{ Bovine liver (NIST 1577c) ${ }^{a}$} \\
\hline $\mathrm{Na}$ & $0,2033 \pm 0,0064$ & $0,1952 \pm 0,0072$ & 3,7 & $0,1923 \pm 0,0148$ & 7,7 \\
\hline K & $1,0230 \pm 0,0640$ & $1,0170 \pm 0,0500$ & 4,9 & $0,9990 \pm 0,0300$ & 3,0 \\
\hline \multicolumn{6}{|c|}{ Meat Homogenate (NIST 1546) ${ }^{\mathrm{b}}$} \\
\hline $\mathrm{Na}$ & $9.990 \pm 716$ & $10.672 \pm 430$ & 4,0 & $10.422 \pm 861$ & 8,3 \\
\hline K & $2.370 \pm 200$ & $2.378 \pm 24$ & 1,0 & $2.373 \pm 151$ & 6,4 \\
\hline
\end{tabular}

a\%: g/100g; ${ }^{\mathrm{pppm}}=\mathrm{mg} \mathrm{kg}^{-1}$. VC: Valor Certificado; VE: Valor Encontrado; RSD: Desvio padrão relativo.

sempre superiores às de $\mathrm{K}$, o que ressalta a importância do desenvolvimento de métodos de análise que possam vir a ser utilizados para o controle de qualidade destes produtos, já que o recomendado é que esses elementos estejam em quantidades semelhantes nos alimentos processados. Das amostras analisadas, a salsicha foi a que apresentou maior concentração de $\mathrm{Na}$, o que pode estar associado ao uso demasiado de sais a base deste elemento em sua composição, como: cloreto de sódio (sabor), nitrito/nitrato de sódio (conservante), fosfato de sódio (estabilizador) e eritorbato de sódio (antioxidante). De acordo com a OMS, ${ }^{21}$ estas amostras apresentaram valores 4 vezes maiores do que o permitido, enquanto que as demais apresentaram concentrações de menor proporção.

\section{Determinação de Mg e Zn em amostras de carnes processadas e frescas}

Para o desenvolvimento dos procedimentos propostos e verificação da concentração dos respectivos analitos, amostras comerciais de carnes processadas e frescas foram preparadas através de microssolubilizações com TMAH e HCOOH (procedimentos 4 e 5, respectivamente). O TMAH é um reagente que tem se apresentado como uma boa alternativa de preparo para amostras biológicas, fornecendo soluções com características de uma suspensão, apresentando um baixo fator de diluição, as quais podem permanecer estáveis por até três meses em temperatura ambiente. Diversos trabalhos atuais reportam que suspensões preparadas em meio de TMAH podem permanecer estáveis por até um ano. ${ }^{12-14}$

A exatidão dos resultados obtidos a partir das metodologias propostas para a determinação de $\mathrm{Mg}$ e $\mathrm{Zn}$ foi avaliada pela análise de duas amostras de referência certificadas de carne, ambas de fígado bovino (NIST 1577b e NIST 1577c, respectivamente). Segundo os resultados apresentados na Tabela 4 , foi possível verificar uma boa concordância entre os resultados obtidos e os valores certificados de concentração para os dois meios de solubilização das amostras. Porém, menores desvios-padrão relativos (RSD) foram obtidos utilizando o TMAH $(\leq 6 \%)$. Além disso, a aplicação do teste $t$-student 
mostrou, para um nível de $95 \%$ de confiança, que os resultados encontrados não apresentaram diferenças significativas em relação aos valores de concentração certificados, o que comprova a exatidão da metodologia proposta.

Como pode ser observado na Tabela 5, concentrações significativas tanto para $\mathrm{Mg}$ como para $\mathrm{Zn}$ foram encontradas nas amostras de carnes analisadas. No entanto, concentrações bem menores de $\mathrm{Zn}$ foram encontradas, o que não desmerece a importância das carnes na alimentação, já que o Zn é um micronutriente essencial que, mesmo estando em baixas concentrações, desempenha diversas funções no organismo. ${ }^{1,4}$ Cabe salientar também que concentrações similares de $\mathrm{Zn}$ em carnes frescas foram encontradas em um trabalho de Andrade et al., ${ }^{3}$ que obtiveram valores de aproximadamente $20 \mathrm{mg} \mathrm{kg}^{-1} \mathrm{de} \mathrm{Zn}$ em amostras bovinas frescas (tipo alcatra).

De acordo com os resultados obtidos para Mg (Tabela 5), as amostras de carnes processadas apresentaram valores de concentrações menores quando comparadas com as amostras de carnes frescas. Estas variações podem ser devidas a diferenças na composição nutricional dos produtos analisados, a dieta dos animais e o solo e/ou tipo de pastagem em que este foi criado. Além disso, pesquisas recentes mostram perdas da qualidade nutricional (vitaminas e minerais) durante as etapas de processamento dos alimentos. ${ }^{2-4,22}$

\section{Parâmetros de mérito}

Os parâmetros de mérito obtidos para as curvas de calibração para $\mathrm{Na}$ e K, tanto por FAAS como por FAES, a partir da metodologia proposta de preparo das amostras por microdigestão (procedimento 1), estão apresentados na Tabela 6. Como pode ser observado, os valores do coeficiente de correlação (R) para ambas metodologias foram superiores a 0,99 , atestando a boa linearidade do método proposto. A faixa linear de trabalho para $\mathrm{Na}$ e $\mathrm{K}$ foi de 0,1 a $2 \mathrm{mg} \mathrm{L}^{-1}$. Os limites de detecção alcançados pelas metodologias são adequados à proposta do trabalho.

A partir dos resultados apresentados na Tabela 7, verifica-se que as curvas de calibração para $\mathrm{Mg}$ e $\mathrm{Zn}$ tanto em meio de TMAH como

Tabela 4. Valores de concentração para Mg e Zn encontrados nos CRMs por FAAS (média \pm desvio; n=3) após solubilização em meio de TMAH ou HCOOH

\begin{tabular}{|c|c|c|c|c|c|}
\hline \multirow{2}{*}{ Amostra } & \multicolumn{3}{|c|}{ TMAH } & \multicolumn{2}{|c|}{$\mathrm{HCOOH}$} \\
\hline & $\mathrm{VC}\left(\mathrm{mg} \mathrm{kg}^{-1}\right)$ & $\mathrm{VE}\left(\mathrm{mg} \mathrm{kg}^{-1}\right)$ & $\operatorname{RSD}(\%)$ & $\mathrm{VE}\left(\mathrm{mg} \mathrm{kg}^{-1}\right)$ & $\operatorname{RSD}(\%)$ \\
\hline \multicolumn{6}{|c|}{ CRM $1577 b$} \\
\hline $\mathrm{Mg}$ & $601 \pm 28$ & $572 \pm 10$ & 1,7 & $589 \pm 40$ & 6,7 \\
\hline $\mathrm{Zn}$ & $127 \pm 16$ & $132 \pm 8$ & 6,0 & $128 \pm 21$ & 16 \\
\hline \multicolumn{6}{|l|}{ CRM $1577 c$} \\
\hline $\mathrm{Mg}$ & $620 \pm 42$ & $634 \pm 36$ & 5,6 & $600 \pm 27$ & 4,5 \\
\hline $\mathrm{Zn}$ & $181,1 \pm 10,0$ & $185,5 \pm 8,0$ & 4,3 & $172,8 \pm 8,0$ & 4,6 \\
\hline
\end{tabular}

VC: Valor Certificado; VE: Valor Encontrado; RSD: Desvio padrão relativo.

Tabela 5. Resultados de concentração encontrados de Mg e Zn nas amostras de carnes em meio de TMAH por FAAS (média \pm desvio; n=3)

\begin{tabular}{|c|c|c|c|c|c|c|}
\hline \multirow{2}{*}{ Amostras } & \multicolumn{3}{|c|}{$\mathrm{Mg}$} & \multicolumn{3}{|c|}{$\mathrm{Zn}$} \\
\hline & $\mathrm{VE}\left(\mathrm{mg} \mathrm{kg}^{-1}\right)$ & $\operatorname{RSD}(\%)$ & $\operatorname{LOD}\left(\mu \mathrm{g} \mathrm{g}^{-1}\right)$ & $\operatorname{VE}\left(\mathrm{mg} \mathrm{kg}^{-1}\right)$ & $\operatorname{RSD}(\%)$ & $\operatorname{LOD}\left(\mu \mathrm{g} \mathrm{g}^{-1}\right)$ \\
\hline Almôndega & $126,84 \pm 3,29$ & 2,6 & & $12,13 \pm 0,86$ & 7,1 & \\
\hline Carne Bovina Processada Enlatada & $120,58 \pm 1,51$ & 1,3 & & $65,25 \pm 4,70$ & 7,2 & \\
\hline Salsicha & $167,03 \pm 9,10$ & 5,4 & 2,40 & $10,28 \pm 0,67$ & 6,5 & 18,4 \\
\hline Carne Bovina in Natura & $215,34 \pm 13,82$ & 6,4 & & $21,94 \pm 1,86$ & 8,5 & \\
\hline Carne Suína in Natura & $253,10 \pm 22,74$ & 8,9 & & $18,11 \pm 0,82$ & 4,5 & \\
\hline
\end{tabular}

VE: Valor Encontrado; RSD = Desvio Padrão Relativo; LOD = Limite de Detecção.

Tabela 6. Parâmetros de Mérito para determinação de Na e $\mathrm{K}$ em amostras de carnes processadas em meio de $\mathrm{HNO}_{3}$ por FAES e FAAS

\begin{tabular}{|c|c|c|c|c|c|c|}
\hline & \multicolumn{3}{|c|}{ FAES } & \multicolumn{3}{|c|}{ FAAS } \\
\hline & $\mathrm{a}\left(\mathrm{L} \mathrm{mg}^{-1}\right)$ & $\operatorname{LOD}\left(\mathrm{mg} \mathrm{g}^{-1}\right)$ & $\mathrm{R}$ & $\mathrm{a}\left(\mathrm{L} \mathrm{mg}^{-1}\right)$ & $\mathrm{LOD}\left(\mathrm{mg} \mathrm{g}^{-1}\right)$ & $\mathrm{R}$ \\
\hline $\mathrm{K}$ & 0,4236 & 0,18 & 0,9998 & 0,5745 & 1,09 & 0,9951 \\
\hline $\mathrm{Na}$ & 0,2416 & 0,20 & 0,9999 & 0,4554 & 1,30 & 0,9993 \\
\hline
\end{tabular}

a: coeficiente angular da curva; LOD: limite de detecção; R: coeficiente de correlação linear.

Tabela 7. Parâmetros de mérito para determinação de Mg e Zn em meio de TMAH e HCOOH para amostras de carnes por FAAS

\begin{tabular}{|c|c|c|c|c|c|c|}
\hline & \multicolumn{3}{|c|}{ TMAH } & \multicolumn{3}{|c|}{$\mathrm{HCOOH}$} \\
\hline & $\mathrm{a}\left(\mathrm{L} \mathrm{mg}^{-1}\right)$ & $\operatorname{LOD}\left(\mu \mathrm{g} \mathrm{g}^{-1}\right)$ & $\mathrm{R}$ & $\mathrm{a}\left(\mathrm{L} \mathrm{mg}^{-1}\right)$ & $\operatorname{LOD}\left(\mu \mathrm{g} \mathrm{g}^{-1}\right)$ & $\mathrm{R}$ \\
\hline $\mathrm{K}$ & 1,8722 & 2,40 & 0,9997 & 1,1763 & 9,40 & 0,9996 \\
\hline $\mathrm{Na}$ & 0,5349 & 18,4 & 0,9984 & 0,3823 & 34,5 & 0,9976 \\
\hline
\end{tabular}

a: coeficiente angular da curva; LOD: limite de detecção; R: coeficiente da curva de calibração. 
em meio de $\mathrm{HCOOH}$, apresentaram bons coeficientes de correlação linear, com $\mathrm{R}>0,99$; bem como mostraram sensibilidades adequadas para atender ao objetivo do trabalho. No entanto, em meio de $\mathrm{HCOOH}$ valores menores de coeficientes angular foram obtidos para ambos elementos, bem como LODs e RSD mais elevados, demonstrando que a utilização de TMAH para solubilização de amostras de carnes se apresentou como uma metodologia mais adequada, sensível e reprodutível. A faixa linear de trabalho para o $\mathrm{Mg}$ foi de 0,1 a 0,4 $\mathrm{mg} \mathrm{L}^{-1}$ e para o $\mathrm{Zn}$ foi de 0,1 a 1,5 $\mathrm{mg} \mathrm{L}^{-1}$. De acordo com os desvios padrão relativos $(\mathrm{RSD} \leq 6,0 \%)$ apresentados na Tabela 4 , verifica-se uma boa reprodutibilidade dos resultados, parâmetro este fundamental para assegurar a confiabilidade das análises.

\section{CONCLUSÃO}

De acordo com os resultados apresentados, pode-se concluir que os métodos de preparo de amostra em microescala são uma alternativa viável para serem aplicados para a determinação de $\mathrm{K}, \mathrm{Mg}, \mathrm{Na}$ e Zn em amostras de carnes. Os métodos propostos são simples e reprodutíveis, exigindo micro quantidades de amostras e reagentes, promovendo de forma eficiente o preparo das respectivas amostras. Além disso, são menos suscetíveis a contaminação, pois permitem a mínima manipulação das amostras e operam em sistemas fechados, sendo adequados para a utilização em análises de rotina.

Os resultados deste estudo ressaltam também a importância do controle de qualidade de $\mathrm{Na}$ e $\mathrm{K}$ em alimentos processados, já que nas amostras reais analisadas foi constatada uma diferença significativa entre as concentrações destes elementos, podendo assim, ser um problema de saúde pública. As altas concentrações de $\mathrm{Na}$ encontradas comprovam a importância de um controle de qualidade, bem como uma redução do teor de sal nos alimentos processados produzidos no Brasil.

\section{AGRADECIMENTOS}

Os autores agradecem ao Conselho Nacional de Pesquisa e Desenvolvimento Tecnológico $(\mathrm{CNPq})$ e à Coordenação de Aperfeiçoamento de Pessoal de Nível Superior (CAPES), agradecem também ao Ministério da Agricultura e Abastecimento (CNPQ/ MAPA/DAS-578261/2008-1) pelo suporte financeiro.

\section{REFERÊNCIAS}

1. Azevedo, F. A., Chasin, A. A. M.; Metais: gerenciamento da toxicidade, Atheneu: São Paulo, 2003.
2. Correia, L. F. M.; Faraoni, A. S.; Pinheiro - Sant'Ana, H. M.; Alim. Nutr. 2008, 19, 83.

3. Andrade, E. C. B.; Barros. A. M.; Mello, V. de S.; Takase, I.; Ciênc. Tecnol. Aliment. 2004, 24, 393.

4. Cozzolino, S.M.F.; Biodisponibilidade de Nutrientes, ed. Manole, Barueri: São Paulo, 2005.

5. Silva, C.; Moneiro, M. L. G.; Ribeiro, R. O. R; Guimarães, C. F. M.; Mano, S. B.; Pardi, H. S.; Mársico, E. T.; Rev. Bras. Ciênc. Vet. 2009, $16,33$.

6. Sofos, J. N.; Food Technol. 1986, 40, 52.

7. Webster, J. L.; Dunford, E. K.; Hawkes, C.; Neal, B. C.; J. Hypertens. 2011, 29, 1043.

8. Ruusunen, M.; Puolanne, E.; Meat Sci. 2005, 70, 531.

9. Anderson, J., Young, L.; Long, E.; Food and Nutrition Series 2008, 9 , 355.

10. Korn, M. G. A.; Boa Morte, E. S.; Santos, D. C. M. B.; Castro, J. T.; Barbosa, J. T. P.; Teixeira, A. P.; Fernandes, A. P.; Welz, B.; Santos, W. P. C.; Santos, E. B. G. N.; App. Spectrosc. Rev. 2008, 43, 67.

11. Krug, F.J.; Métodos de Preparo de Amostras: Fundamentos sobre preparo de amostras orgânicas e inorgânicas para análise elementar, 1 ed., Edição do autor, Piracicaba: São Paulo, 2010.

12. Nóbrega, J. A.; Santos, M. C.; Sousa, R. A.; Cadore, S.; Barnes, R. M.; Tatro, M.; Spectrochim. Acta Part B. 2006, 61, 465.

13. Martins. P.; Pozebon. D.; Dressler, V.L.; Kemieciki G.A.; Anal. Chem. Acta. 2002, 470, 195.

14. Ribeiro, A. S.; Curtius, A. J.; Pozebon, D.; Microchem. J. 2000, 64, 105.

15. Mortari, S.; Saidelles, A. P. F.; Barin, J. S.; Flores, É. M. M.; Martins, A. F.; Microchim. Acta. 2004, 148, 157.

16. Campos, R.C.; Vegueria, S. F. J; Gonçalves, R. A.; Wagener, A. L. R.; J. Braz. Chem. Soc. 2009, 20, 1925.

17. Rosini, F.; Nascentes, C. C.; Nóbrega, J. A.; Quim. Nova. 2004, 27, 1012.

18. Scriver, C.; Kan, M.; Willie, S.; Soo, C.; Birnboim, H.; Anal. Bioanal. Chem. 2005, 381, 1460.

19. Bond, A. M.; Canterford, D. R.; Anal. Chem. 1971, 43, 134.

20. Brasil. Ministério da Agricultura Pecuária e Abastecimento. Método para análise de microelementos por GF AAS, N 400/03, emissão 1985, revisão 2005.

21. World Health Organization. Strategies to monitor and evaluate population sodium consumption and sources of sodium in the diet. Report of a joint technical meeting convened by WHO and Government of Canada. Canada, Oct, 2010.

22. Mistura, L. P. F.; Colli, C.; Ciênc. Tecnol. Aliment. 2009, 29, 195. 